\title{
First Canadian experience with robotic laparoendoscopic single-site vs. standard laparoscopic living-donor nephrectomy: A prospective comparative study
}

\author{
Patrick P. Luke ${ }^{1,3,4}$; Shahid Aquil ${ }^{1,2,3}$; Bijad Alharbi ${ }^{1,2,3}$; Hemant Sharma ${ }^{1,2,3}$; Alp Sener ${ }^{1,3-5}$ \\ ${ }^{1}$ Department of Surgery, Division of Urology; ${ }^{2}$ Western University Schulich School of Medicine and \\ Dentistry; ${ }^{3}$ Multi-Organ Transplant Program, London Health Sciences Centre; ${ }^{4}$ Matthew Mailing Centre for \\ Translational Transplant Studies, London Health Sciences Centre; ${ }^{5}$ Department of Microbiology and Immunology; \\ Western University, London, ON, Canada
}

Cite as: Can Urol Assoc J 2018 June 8; Epub ahead of print. http://dx.doi.org/10.5489/cuaj.5275

Published online June 8, 2018

$* * *$

\section{Abstract}

Introduction: We aimed to compare the outcomes of robotic laparoendoscopic single-site living donor nephrectomy (R-LESS LDN) vs. standard laparoscopic living donor nephrectomy (LLDN).

Methods: Between October 2013 and November 2015, 39 patients were allocated to either standard laparoscopic living donor nephrectomy (LLDN; $n=25)$ or robotic-assisted laparoendoscopic single-site living donor nephrectomy (R-LESS LDN; n=14). Patient demographics, perioperative outcomes, analgesic requirement, visual analogue scale of pain at postoperative days $1,3,7$, and 30 , and a health-related quality of life and body image questionnaire were prospectively collected.

Results: There were no significant differences in demographics and intraoperative outcomes between the two cohorts. The R-LESS LDN cohort had lower analgesic requirement $(\mathrm{p}=0.002)$ and lower visual pain scores on days 1 and $3(\mathrm{p}=0.001)$. Additionally, body image and satisfaction scores in the R-LESS group were also superior compared to the LLDN cohort $(\mathrm{p}=0.008)$. There was no significant difference in the postoperative complications according to the Clavien-Dindo system. Recipient graft functional outcomes were equivalent.

Conclusions: This is the first evidence that R-LESS LDN is safe and associated with comparable surgical and early functional outcomes compared to LLDN, while pain, donor body image, and satisfaction scores were improved compared to LLDN. 


\section{Introduction}

Kidney transplantation is the preferred option for patients with end stage renal disease. However due to shortage of donor organ, we turn to living donor kidney transplants which provide better graft function and survival compared to organs from deceased donors. [1,2]. Historically, living donor nephrectomy (LDN) had been performed as an open technique which brought significant comorbidity and impacted donor quality of life. Over the last two decades, surgical practices have developed with the aim of improving post-operative donor recovery, while maintaining surgical quality; these modifications to the donor operation have included mini-incision musclesplitting open LDN through a dorsal lumbotomy [3], to minimally invasive techniques, including standard laparoscopy [4], hand-assisted laparoscopy [5] and retroperitoneoscopy [6]. The advent of laparoscopic LDN was not only associated with a significant rise in the number of living donors globally, but also had a major impact on patient satisfaction with the operation and improved post-surgical recovery and pain scores. More recently, novel minimally invasive techniques have been introduced including laparoendoscopic single-site surgery (LESS) [7], natural orifice transluminal endoscopic surgery (NOTES)-assisted laparoscopy [8,9], minilaparoscopy [10], and robot-assisted laparoscopy [11]; all of which have been applied to living donor surgery.

With surgical technologies advancing towards less invasive methods and with increasing pressure from patients to incorporate these new techniques into practice, data has emerged suggesting that single incision surgery may be the next major advance to the living donor operation. In a recent systematic review and meta-analysis of over 1500 cases comparing laparoscopic and LESS nephrectomy, Autorino et al. [12,13,14] showed that LESS patients benefited from decreased postoperative pain, lower analgesic requirements, shorter hospital stay, faster recovery time, and not surprisingly, a better cosmetic outcome. As we want to minimize the burden of living donor surgery to the healthy young, active individual, the concept of minimizing the skin incision is appealing and may further incentivize organ donation.

The learning curve of LESS donor nephrectomy procedure is notoriously steep, even in experienced centers. We predicted that the use of robotic assistance would make a significant impact on the learning curve of the LESS surgery as it offers a significant improvement in visualization, and intracorporeal maneuverability and dexterity. Therefore, we hypothesized that robotic assisted LESS living donor surgery(R-LESS) is feasible, with minimal impact upon operative time and complications. Furthermore, we evaluated whether R-LESS has a positive impact upon patient outcomes, pain, and quality of life following living donation.

\section{Methods}

Between October 2013 and November 2015, 46 patients underwent living donor nephrectomy at University Hospital, London Health Sciences Center, London, Canada. Consecutive patients were approached at the time of their first clinic assessment to be entered into the prospective study (REB\#101769). All patients were consented to receive either standard laparoscopic donor 
nephrectomy or robot assisted LESS nephrectomy, randomization was not possible as access to the robot was not predictable. All donor surgeries were performed by two surgeons (Patrick Luke, Alp Sener). One surgeon had experience with R-LESS pyeloplasty [18], nephrectomy, partial nephrectomy, adrenalectomy prior to performing donor nephrectomy. The donors were slotted for a standing date, if the robot was available that day, the patient was placed in the RLESS arm, if the robot was not available, the patient was slotted for the LLDN arm of the study. Seven patients decided not to participate. Patients underwent either standard laparoscopic living donor nephrectomy (LLDN; $n=25$ ) or robotic assisted laparoendoscopic single-site living donor nephrectomy (R-LESS LDN; n=14). Donor and recipient demographic characteristics were collected including: age, gender, body mass index (BMI), side of procedure and number of renal arteries. Perioperative outcomes between the two procedures were compared using operative time, warm ischemia time (WIT), estimated blood loss, hospital length of stay, analgesic requirements (calculated in terms of hydromorphone equivalents), visual analogue scale [15] of pain on post-operative days 1, 3, 7, and 30 days post-operatively. Graft function based on serum creatinine and MDRD based eGFR measurements on day 3 and one year, and a health-related quality of life and body image questionnaire [16] were also performed on days 3, 7 and 30 post donation. Delayed graft function (DGF) was defined as the need for hemodialysis in the first week following transplantation. Postoperative complications were graded according to the Clavien-Dindo system [17].

\section{Donor nephrectomy surgical technique}

Robot-assisted laparoendoscopic single-site technique

The da Vinci Si Surgical System (Intuitive Surgical, Sunnyvale CA, USA) was used for all procedures. Patients were positioned in the right lateral decubitus position. A single incision was made through the umbilicus measuring approximately $4.0 \mathrm{~cm}$ in length and a GelPort (Applied Medical, Rancho Santa Margarita, CA, USA) pre-punctured with four trocars (camera port, two $8 \mathrm{~mm}$ robot working trocars, and a $10 \mathrm{~mm}$ accessory trocar) was placed through the umbilicus (Figure. 2). Once the abdomen was insufflated, the daVinci robot was docked behind the shoulder of the patient with the first setup joint locked in a straight position in order to facilitate proper insertion of the working instruments as previously described [18] (Figure. 1).

The operation began with mobilization of the descending colon. The ureter was then identified and circumferentially dissected along the gonadal vein. Following the left gonadal in the cephalad direction, the left renal vein was subsequently identified. Gerota's fascia was incised, and the kidney was separated from its attachments to the left adrenal gland and the spleen. The renal vein was circumferentially dissected. The gonadal vein was divided close to the renal vein between clips. The left renal artery was circumferentially isolated to the level of its aortic takeoff. The robot was then undocked and the ureter was divided using $10 \mathrm{~mm}$ Hemolock clips. After replacing the $10 \mathrm{~mm}$ port with a $15 \mathrm{~mm}$ port (Ethicon Endosurgery, Cincinnati OH, 
USA), the renal artery and vein sequentially controlled and stapled using a 35mm endovascular stapler (Ethicon, Cincinnati OH, USA).

The kidney was then retrieved with a $15 \mathrm{~cm}$ Endocatch bag (Ethicon Endosurgery, Cincinnati OH, USA) placed through the $15 \mathrm{~mm}$ port and retrieved through the GelPort. The kidney was immediately flushed with Custodiol HTK solution (Odyssey pharmaceuticals Inc., Florham park, NJ, USA) containing 10,000 IU of heparin until the effluent was clear and placed on ice until transplantation.

$\underline{\text { Standard multi-port laparoscopic donor nephrectomy }}$

A standard laparoscopic donor nephrectomy was carried out in the usual fashion [18]. Once the patient was positioned in the manner described above, a Hasson blunt tipped trochar was inserted under direct vision just lateral and superior to the umbilicus; this was used as the camera port. Once the abdomen was insufflated, a $5 \mathrm{~mm}$ trochar below the costal margin and a 10-12mm trochar was inserted in the lower quadrant. Standard instruments were utilized in all cases. At the point of organ extraction, we made a Pfannenstiel incision and introduced a $15 \mathrm{~mm}$ Endocatch bag for the retrieval, which was done in a similar fashion as described above.

In both groups, warm ischemic time was defined as the time between initial stapler application to the renal artery and hypothermic organ perfusion with preservation solution.

\section{Statistical analyses}

Demographic characteristics, perioperative and postoperative outcomes were compared between each of the two groups. Complications were classified according to the Clavien-Dindo system. Internal reliability for each scale was evaluated by Cronbach's alpha. The Cronbach's alpha for the body image scale was 0.82 and Cronbach's alpha for the Cosmetic questionnaire was 0.76 . These values suggest that the scales showed good internal consistency. Mean scale scores for the of each scale at day 1,3,7 and 30 post-operative day were evaluated for significance of difference using the non-parametric Wilcoxon signed-rank test for hypothesis testing of repeated measurements. The categorical data for assessing differences in both R-LESS LDN group and LLDN group were analyzed using a paired Student's t- test. Data analyses were performed using SPSS version 22.0 (SPSS, Inc. Chicago, IL, USA).

\section{Results}

The demographic characteristics, intraoperative and postoperative outcomes for patients in both cohorts are listed in Table 1 and 2. There were no significant differences in intraoperative outcomes including operative time between LLDN and R-LESS LDN. Although there were no conversions of R-LESS to LLDN or open DN, the addition of a $5 \mathrm{~mm}$ port was required via the GelPort to facilitate R-LESS in 4 cases where the spleen retraction was difficult. We observed no delayed graft function in both group and no statistical differences in the mean serum creatinine of the recipients at one-year post transplant. Mean creatinine of donors was equivalent at day 3 in both cohorts. The R-LESS cohort had statistically superior visual pain scores on day 1 and 3 
$(\mathrm{p}<0.001)$ and the mean hydromorphone equivalent analgesia needed in the R-LESS cohort was $15.9 \pm 3.3 \mathrm{mg}$ in first 48hrs after surgery compared to $18.15 \pm 5.1 \mathrm{mg}$ for LLDN cohort $(\mathrm{p}=0.002)$.

In R-LESS LDN group, one patient developed a retroperitoneal hematoma that was treated conservatively and transfused with two units of packed red blood cells. In LLDN group, two patients developed abdominal wall hematomas (managed conservatively), two patients developed neuromuscular pain with bilateral flank discomfort and one patient with a BMI of 38 developed a port site infection. Overall, postoperative complication rates between the two cohorts were low, with all complications classified as Grade 2 according to the Clavien-Dindo system.

All 14 patients who underwent R-LESS LDN and all 25 who underwent LLDN responded to The Body Image Scale, Cosmesis Scale and Visual Analog Pain Scale questionnaires. The responses are shown in Tables 3-7.

\section{Body image and cosmesis following living donation}

The first question asked of the donors was "Are you less satisfied with your body since the operation?” In the R-LESS group, 92\% of respondents reported complete satisfaction by day 3, whereas only $50 \%$ of respondents in the laparoscopic group reported complete satisfaction by the third day ( $\mathrm{p}=0.002)$. Although this persisted up to 7 days, by post-operative day 30, both groups had similar satisfaction scores ( $\mathrm{p}=0.71$, Table 3 ). In response to second question "Do you think the operation has damaged your body? “, on days 3 and 7, patients undergoing R-LESS felt they had no or little damage to their body compared to laparoscopic group ( $\mathrm{p}<0.001$ ) but by day 30 , both groups reported similar findings ( $p=0.51$, Table 4$)$. The final question in the body image index was “Is it difficult to look at yourself naked as a result of the operation?” Both groups reported similar responses to this question at all-time points on post-operative days 3, 7 and 30, respectively ( $p=0.41$, Table 5 ). With respect to cosmesis, patients were asked to rate their satisfaction with their surgical scar on a scale from 1-7. Cosmetically, living donors were more satisfied with their incisions in the R-LESS group compared to the LLDN group across all time points on post-operative days 3, 7 and 30. ( $p=0.008$, Table 6, Figure. 3).

Visual analog pain score

Living donors from each cohort were asked how they rated their pain on a visual analog pain scale from 1-10 on post-operative days 1, 3, 7 and 30. The R-LESS cohort had statistically superior pain scores (none/mild pain R- LESS 50\% vs. 26\% LLDN group) on day 1 and 3 $(\mathrm{p}<0.001)$, however from day 7 onwards, both groups showed similar perceptions of pain and clinically not significant. ( $\mathrm{p}=0.16$, Table 7$)$. 


\section{Discussion}

With the increasing number of patients requiring kidney transplantation, multiple strategies to increase the number of donors, including laparoscopic living donation, have been developed. Advancements in laparoscopy have led to the use of LESS surgery in living donation, unfortunately, the ergonomics of single port surgery lends itself to a very steep learning curve. The current study was designed to evaluate whether a single incision robotic platform would allow surgeons to make the leap to single incision living donor surgery. We are the first to demonstrate that there were no significant differences in intraoperative outcomes between LLDN and R-LESS-LDN cohorts. Analysis showed statistically differences in visual analogue pain scores on day 1 , and $3(\mathrm{p}<0.001)$ between the R-LESS LDN and LLDN cohorts however from day 7 onwards, both groups showed similar perceptions of pain and clinically not significant. $(p=0.16)$. In addition, the analgesic requirements were lower for R-LESS cohort $(p=0.002)$ immediately after surgery. They demonstrate earlier improvement in the donor body image and satisfaction in R-LESS LDN cohort had a high satisfaction score compared to the LLDN cohort $(p=0.008)$. There were no significant differences in the post-operative complication rates in either cohort.

Gill et al. [7] first reported the successful completion of four single-port transumbilical LDNs. Soon after, the same group reported the first retrospective matched pair comparison of LESS LDN to standard LDN, concluding that the LESS approach may be associated with quicker convalescence and comparable early allograft outcomes [19]. Since then, other groups have reported comparative assessments of the two LDN approaches, with conflicting findings [20-22]. When adopting a novel surgical technique, patient safety represents a key factor and this is especially true in the case of a LDN. As a general principle, all eligible laparoscopicsurgery patients may be considered for LESS. At the same time, patient selection with LESS must be more rigorous to minimize the surgical risk. R-LESS experience into Gill but no comparison versus laparoscopic [7]. In our study, we found no significant differences in total operating time between R-LESS LDN including robot set-up time compared with LLDN $(\mathrm{p}=0.90)$. Operating time is routinely considered as a parameter to estimate the surgical learning curve. In this regard, Stamatakis et al. [23] observed a little change over the course of their series, suggesting a very shallow learning curve, suggesting that for a surgeon already experienced with LDN, LESS LDN case numbers might not be as important in determining operating times after a plateau has been reached. Both surgeons in our cohort have extensive expertise in laparoscopic surgery with one having experience in R-LESS surgery, mainly with pyeloplasty [18]. The experience provided safety for donor but limiting ability to assess learning curve for R-LESS.

WIT is traditionally recognized as a surrogate measure of surgical quality during LDN [27]. We did not find a significant difference in WIT between the two cohorts $(p=0.52)$. In their comparative studies, Canes et al. [19] found WIT to be twice as long in the LESS LDN group, and most of this extra time was spent creating an adequate fascial incision, as this site was not be 
prepared before extraction. In contrast, Stamatakis et al. [23] were the first to document a statistically significant decrease $(0.5 \mathrm{~min})$ in WIT with LESS LDN as compared with LDN. The authors attributed this finding to the use of the GelPOINT ${ }^{\mathrm{TM}}$, eliminating the need to complete an incision after transection of the renal vasculature. Our technique of using the GelPort for access allowed for a larger facial incision which enabled quick extraction of the kidney while still maintaining a smaller skin incision through the umbilicus. More clinically relevant than WIT is graft function, which was assessed only in few of the studies by using creatinine levels [19, 20]. In the present study, we demonstrate that serum creatinine and eGFR levels were similar between the two groups $(\mathrm{p}=0.51)$, even at one year post-transplant. In addition, we found no delayed graft function in either group, which is a strong predictor of early graft injury and poor longer term function [28]. Thus further supporting that the R-LESS approach to living donation does not compromise graft outcomes.

It is a well-established principle that single renal artery left kidneys are preferred by most transplant surgeons due to the longer length of the left renal vein compared with the right renal vein. In the present study, all of the R-LESS group had left sided nephrectomies. We did not exclude right donor nephrectomy intentionally. More importantly, we had selected the left side despite supernumery arteries even before the case was assigned to the R-LESS cohort, suggesting that multiple vessels can also be handling with the R-LESS approach and that these patients should not be excluded. In other reported studies, some investigators considered only left-sided donors [19, 21] and non-complex vasculature [21, 25] as inclusion criteria for LESS donation, whereas others did not [20,21]. As excellent long-term outcomes can be obtained with LDN with right and/or complex vascular anatomy requiring reconstruction, the presence of multiple renal arteries should ideally not preclude R-LESS kidney donation [28], at least in experienced centers.

The rationale behind the adoption of LESS is mainly based on the potential gain for the patient in terms of lower postoperative pain, shorter hospital stay, and ultimately faster recovery. Length of stay represents an unreliable endpoint in this patient population, as donors may express the desire to remain in the hospital longer because of psychosocial considerations [12]. In present study analysis showed statistically differences in visual analogue pain scores on day 1 , and $3(p<0.001)$ between the R-LESS LDN and LLDN cohorts as well as in analgesic requirements, however from day 7 onwards, both groups showed similar perceptions of pain and clinically not significant. $(\mathrm{p}=0.16)$. They demonstrate earlier improvement in the donor body image and satisfaction in R-LESS LDN cohort had a high satisfaction score compared to the LLDN cohort ( $\mathrm{p}=0.008$ ). However, there was no significant difference of the hospital stay between R-LESS LDN compared with LLDN ( $\mathrm{p}=0.81$ ). In keeping with our findings, Fan et al. [12] also reported reduced postoperative pain, and lower analgesic requirement for LESS nephrectomy procedures. This is in congruence with what we observed and is expected with smaller total length of incisions in the R-LESS cohort. 
Complication rates are broadly considered surrogate markers for surgical complexity. Accurate reporting of complications is important for preoperative counselling, and for identifying modifiable risk factors to decrease complication rates. Greco et al. [30] investigated risk factors for complications in a multi-institutional series of LESS surgery for a range of upper urinary tract disease and found an overall complication rate of 17\%. In a larger analysis of surgical outcomes from LESS cases of mixed indications, Autorino et al. [29] reported a 9.4\% postoperative overall complication rate, most of them being of low Calvien grade. In the present analysis, we did not find any significant difference in terms of postoperative complication rates between the two cohorts, with all complications being Grade 2 according to the Clavien-Dindo classification.

Although not randomized, this is the first detailed prospective assessment of the R-LESS technique in performing LDN. Although our numbers are small, we publish these results as an indicator of quality for our novel technique and demonstrate modest but significant benefits in this population of young healthy patients, who may engender benefit from a cosmetically superior operation. A limitation was that we were not able to blind the patients immediately preop or post-op (with abdominal binders to mask the scars), however this may be what is needed to truly create a randomized trial in the future. However, we did not emphasize that one technique was more beneficial than the other during the consent process so as to minimize patient bias. It has been our hope that the minimally invasive nature of this technique would increase the appetite for healthy active individuals to participate in living donation. In fact, it is of interest that the availability of R-LESS technique has been associated with an increased interest in living donation by potential donor patients at our institution. Furthermore, we hope that our excellent preliminary results will encourage other groups to assess R-LESS donor nephrectomy as a part of multi-centred prospective study to firmly establish the procedure as a reasonable option for donor nephrectomy. With the advancement of robotic platforms intended for single incision surgery by companies such as Titan Medical ${ }^{\mathrm{TM}}$ and Intuitive Surgical ${ }^{\circledR}$, the role of robotic surgery in donor nephrectomy should be re-assessed on a continuous basis.

\section{Conclusion}

These are the first reported results demonstrating that R-LESS LDN procedure offers comparable surgical and early functional outcomes compared to standard LDN but with reduced postoperative pain, improved body image scores and overall satisfaction with the donation process. Robotic LESS is more technically challenging than standard LLDN counterpart. However, with increasing level of expertise in users across many centers and the continually advancing technology in robotics, this novel approach should be compared with standard LLDN in a well-designed, large, prospective, randomized, multi-center study before gaining wider acceptance. 


\section{References}

1. Louis G, Hubert J, Ladriere M et al: Robotic-assisted laparoscopic donor nephrectomy for kidney transplantation. An evaluation of 35 procedures. Nephrol Ther 2009; 5: 623.

2. Cecka JM. The UNOS Scientific Renal Transplant Registry- 2000. Clin Transpl 2000; 18.

3. Antcliffe D, Nanidis TG, Darzi AW et al: A meta-analysis of mini-open versus standard open and laparoscopic living donor nephrectomy. Transpl Int 2009; 22: 463-74.

4. Ratner LE, Ciseck LJ, Moore RG et al: Laparoscopic live donor nephrectomy. Transplantation 1995; 60: 1047-9.

5. Wadström J, Martin AL, Estok R et al: Comparison of hand-assisted laparoscopy versus open and laparoscopic techniques in urology procedures: a systematic review and metaanalysis. J Endourol 2011; 25: 1095-104.

6. Bachmann A, Wolff T, Ruszat R et al: Retroperitoneoscopic donor nephrectomy: a retrospective, non-randomized comparison of early complications, donor and recipient outcome with the standard open approach. Eur Urol 2005; 48: 90-6.

7. Gill IS, Canes D, Aron $\mathrm{M}$ et al: Single port transumbilical (E-NOTES) donor nephrectomy. J Urol 2008; 180: 637-41.

8. Alcaraz A, Musquera M, Peri L et al: Feasibility of transvaginal natural orifice transluminal endoscopic surgery-assisted living donor nephrectomy: is kidney vaginal delivery the approach of the future? Eur Urol 2011; 59: 1019-25.

9. Kaouk JH, Khalifeh A, Laydner $\mathrm{H}$ et al: Transvaginal hybrid natural orifice transluminal surgery robotic donor nephrectomy: first clinical application. Urology 2012; 80: 1171-5.

10. Breda A, Villamizar JM, Faba OR et al: Laparoscopic live donor nephrectomy with the use of 3-mm instruments and laparoscope: initial experience at a tertiary center. Eur Urol 2012; 61: 840-4.

11. Renoult E, Hubert J, Ladrière $\mathrm{M}$ et al: Robot-assisted laparoscopic and open live-donor nephrectomy: a comparison of donor morbidity and early renal allograft outcomes. Nephrol Dial Transplant 2006; 21: 472-7.

12. Fan X, Lin T, Xu K et al: Laparoendoscopic single-site nephrectomy compared with conventional laparoscopic nephrectomy: a systematic review and meta-analysis of comparative studies. Eur Urol 2012; 62: 601-12.

13. Riccardo Autorino, Luis Felipe Brandao, Bashir Sankari et al: Laparoendoscopic singlesite (LESS) vs laparoscopic living-donor nephrectomy: a systematic review and metaanalysis. BJU Int 2015; 115: 206-215.

14. Lee Richstone, Soroush Rais-Bahrami, Nikhil Waingankar at al: Pfannenstiel laparoendoscopic single-site (LESS) vs conventional multiport laparoscopic live donor nephrectomy: a prospective randomized controlled trial. BJU Int 2013; 112, 616-622. 
15. H. Breivik; P. C. Borchgrevink; S. M. Allen et al: Assessment of Pain Intensity and Pain Relief in Acute Pain. Br J Anaesth. 2008; 101(1):17-24.

16. Thomas Chin, D. Nikitin, B. Nibhanupudy et al: Initial Experience with Single Incision Laparoscopic Donor Nephrectomy. American Journal of Transplantation, 9 (S2), A859, p48, 2009.

17. Dindo D, Demartines N, Clavien PA. Classification of surgical complications: 398 a new proposal with evaluation in a cohort of 6336 patients 399 and results of a survey. Ann Surg 2004; 240: 205-13.

18. Jeffrey Law, Alp Sener, Patrick P. Luke et al: First Canadian experience with robotic single-incision pyeloplasty: Comparison with multi-incision technique. Can Urol Assoc J 2016; 10(3-4):83-8.

19. Canes D, Berger A, Aron $M$ et al: Laparo-endoscopic single site (LESS) versus standard laparoscopic left donor nephrectomy: matched-pair comparison. Eur Urol 2010; 57: 95101.

20. Afaneh C, Aull MJ, Gimenez E et al: Comparison of Laparoendoscopic single-site donor nephrectomy and conventional laparoscopic donor nephrectomy: donor and recipient outcomes. Urology 2011; 78: 1332-7.

21. Lunsford KE, Harris MT, Nicoll KN et al: Single-site laparoscopic living donor nephrectomy offers comparable perioperative outcomes to conventional laparoscopic living donor nephrectomy at a higher cost. Transplantation 2011; 91: e16-7.

22. Andonian S, Rais-Bahrami S, Atalla MA et al: Laparoendoscopic single-site Pfannenstiel versus standard laparoscopic donor nephrectomy. J Endourol 2010; 24: 429-32.

23. Stamatakis L, Mercado MA, Choi JM et al: Comparison of laparoendoscopic single site (LESS) and conventional laparoscopic donor nephrectomy at a single institution. BJU Int 2013; 112: 198-206.

24. Barth RN, Phelan MW, Goldschen L et al: Single-port donor nephrectomy provides improved patient satisfaction and equivalent outcomes. Ann Surg 2013; 257: 527-33.

25. Kurien A, Rajapurkar S, Sinha L et al: First prize: standard laparoscopic donor nephrectomy versus laparoendoscopic single-site donor nephrectomy: a randomized comparative study. J Endourol 2011; 25: 365-70.

26. Richstone L, Rais-Bahrami S, Waingankar N et al: Pfannenstiel laparoendoscopic singlesite (LESS) vs conventional multiport laparoscopic live donor nephrectomy: a prospective randomized Controlled trial. BJU Int 2013; 112: 616-22.

27. Hellegering J, Visser J, Kloke HJ et al: Deleterious influence of prolonged warm ischemia in living donor kidney transplantation. Transplant Proc 2012; 44: 1222-6.

28. Abouchacra S, Chaaban A, Hakim R et al: Renal biomarkers for assessment of kidney function in renal transplant recipients: how do they compare? Int Urol Nephrol 2012; 44:1871-6. 
29. Autorino R, Kaouk JH, Yakoubi R et al: Urological Laparoendoscopic single site surgery: multi-institutional analysis of risk factors for conversion and postoperative complications. J Urol 2012; 187: 1989-94.

30. Greco F, Cindolo L, Autorino R et al: Laparoendoscopic single-site upper urinary tract surgery: assessment of postoperative complications and analysis of risk factors.Eur Urol 2012; 61: 510-6. 


\section{Figures and Tables}

Fig. 1. (A) Positioning of the robot over the posterior shoulder of the patient for single-incision surgery. The patient is positioned at 45 degrees in the right lateral oblique position. (B) The first setup joint is locked in a straight position to facilitate proper insertion of multiple working instruments in the umbilical port. Rendering (C) Intuitive Surgical 2015 with permission.
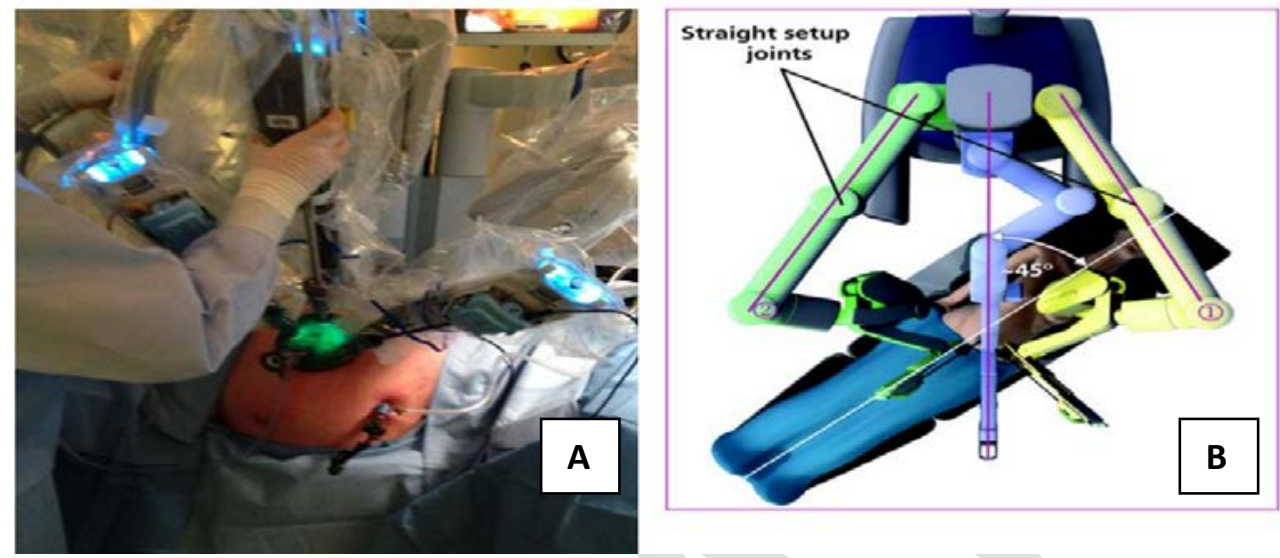

Fig. 2. (A) Placement of inner phalange using GelPort device. (B) Disposable and $8 \mathrm{~mm}$ robotic ports placed through the GelPort device.

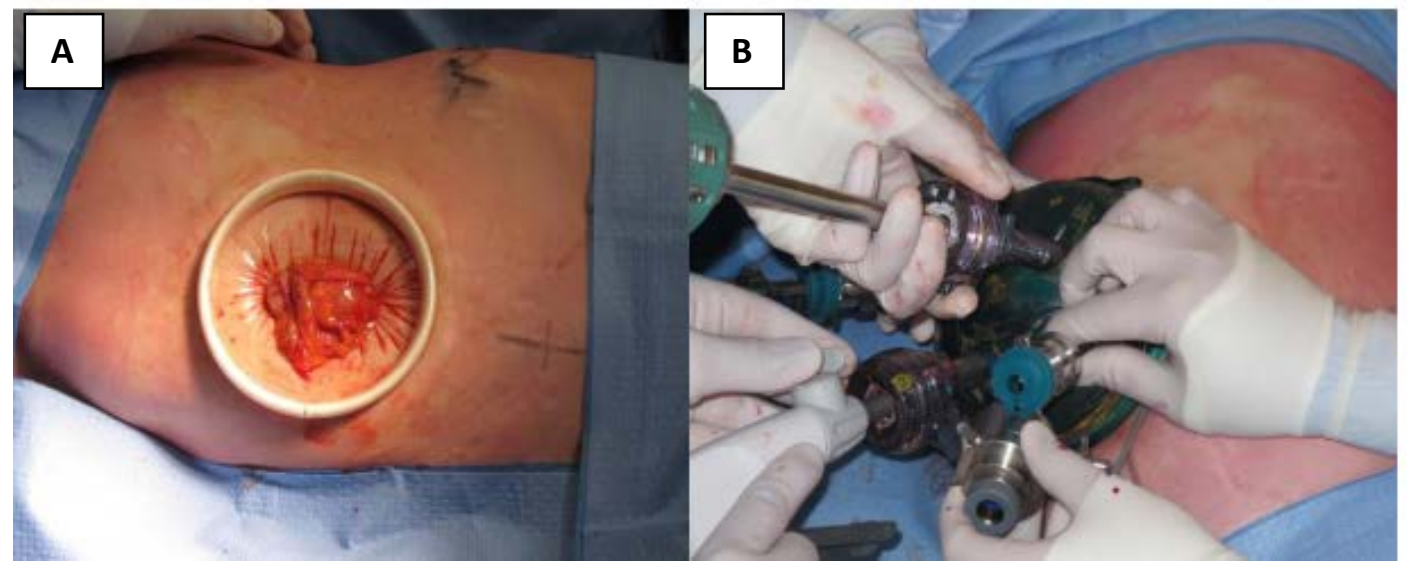




\begin{tabular}{|l|c|c|c|}
\hline \multicolumn{4}{|l|}{ Table 1. Demographic characteristics } \\
\hline & \multicolumn{2}{|c|}{ Type of donor surgery } \\
\hline & Laparoscopic & R-LESS & p \\
\hline Number of patients & 25 & 14 & - \\
\hline Age, years (range) & $50(26-68)$ & $51(41-64)$ & 0.97 \\
\hline Male: Female & $7: 18$ & $9: 5$ & - \\
\hline BMI $\left(\mathrm{kg} / \mathrm{m}^{2}\right)$ & $27.1 \pm 3.8$ & $25.8 \pm 3.4$ & 0.24 \\
\hline Right: Left & $5: 20$ & $0: 14$ & - \\
\hline Multiple arteries & 4 & 3 & - \\
\hline
\end{tabular}

Data are presented as mean \pm standard deviation. BMI: body mass index. R-LESS: robotic laparoendoscopic single-site.

\begin{tabular}{|c|c|c|c|}
\hline \multicolumn{4}{|c|}{$\begin{array}{l}\text { Table 2. Intraoperative and postoperative outcomes in patients undergoing la } \\
\text { vs. robot-assisted LESS donor nephrectomy } \\
\\
\text { Type of donor surgery }\end{array}$} \\
\hline & Laparoscopic & R-LESS & $\mathrm{p}$ \\
\hline Number of patients & 25 & 14 & - \\
\hline Operative time (min) & $240 \pm 53$ & $269 \pm 75$ & 0.90 \\
\hline Estimated blood loss (mL) & $103 \pm 70$ & $140 \pm 59$ & 0.24 \\
\hline Length of stay (days) & $3.5 \pm 0.86$ & $3.1 \pm 0.70$ & 0.81 \\
\hline WIT (min) & $4.15 \pm 1.1$ & $4.3 \pm 1.1$ & 0.52 \\
\hline Total hydromorphone equivalent (mg) & $18.15 \pm 5.1$ & $15.9 \pm 3.3$ & 0.002 \\
\hline Serum creatinine $(\mu \mathrm{mol} / \mathrm{L}$, Day 3$)$ & $96 \pm 10$ & $95 \pm 15$ & 0.51 \\
\hline Serum creatinine ( $\mu \mathrm{mol} / \mathrm{L}, 1$ year) & $102 \pm 21$ & $106 \pm 12$ & 0.22 \\
\hline eGFR (mL/min per $\left.1.73 \mathrm{~m}^{2}\right)$ & $84 \pm 17.14$ & $86 \pm 17.74$ & 0.51 \\
\hline DGF & 0 & 0 & - \\
\hline \multicolumn{4}{|l|}{ Complications: (Clavien-Dindo) } \\
\hline Hematoma requiring transfusion & 0 & 1 & - \\
\hline Abdominal wall hematoma no transfusion & 1 & 0 & - \\
\hline Neuromuscular pain & 2 & 0 & - \\
\hline Port site infection & 1 & 0 & - \\
\hline
\end{tabular}

All complications classified as Grade 2 according to the Clavien-Dindo system. Data are presented as mean \pm standard deviation. eGFR: estimated glomerular filtration rate; DGF: delayed graft function; R-LESS: robotic laparoendoscopic single-site; WIT: warm ischemic time. 
Laparoendoscopic single-site vs. standard living donor nephrectomy

\begin{tabular}{|c|c|c|c|c|}
\hline & & & pera & \\
\hline & & 3 & 7 & 30 \\
\hline & Yes, extremely & $0 \%$ & $0 \%$ & $8 \%$ \\
\hline I ECS nobati & Quite a bit & $0 \%$ & $8 \%$ & $0 \%$ \\
\hline LESS RODOUC & A little bit & $8 \%$ & $34 \%$ & $34 \%$ \\
\hline & No, not at all & $92 \%$ & $58 \%$ & $58 \%$ \\
\hline & Yes, extremely & $0 \%$ & $4 \%$ & $0 \%$ \\
\hline I anarncronic & Quite a bit & $0 \%$ & $4 \%$ & $0 \%$ \\
\hline Ldpatoscopic & A little bit & $50 \%$ & $35 \%$ & $26 \%$ \\
\hline & No, not at all & $50 \%$ & $57 \%$ & $74 \%$ \\
\hline
\end{tabular}

R-LESS group, 92\% of respondents reported complete satisfaction by day 3, whereas only 50\% of respondents in the LLDN group reported complete satisfaction by the third day ( $p=0.002)$, but by day 7 and 30, both groups had similar satisfaction scores $(p=0.71)$. LLDN: laparoscopic living donor nephrectomy; R-LESS: robotic laparoendoscopic single-site.

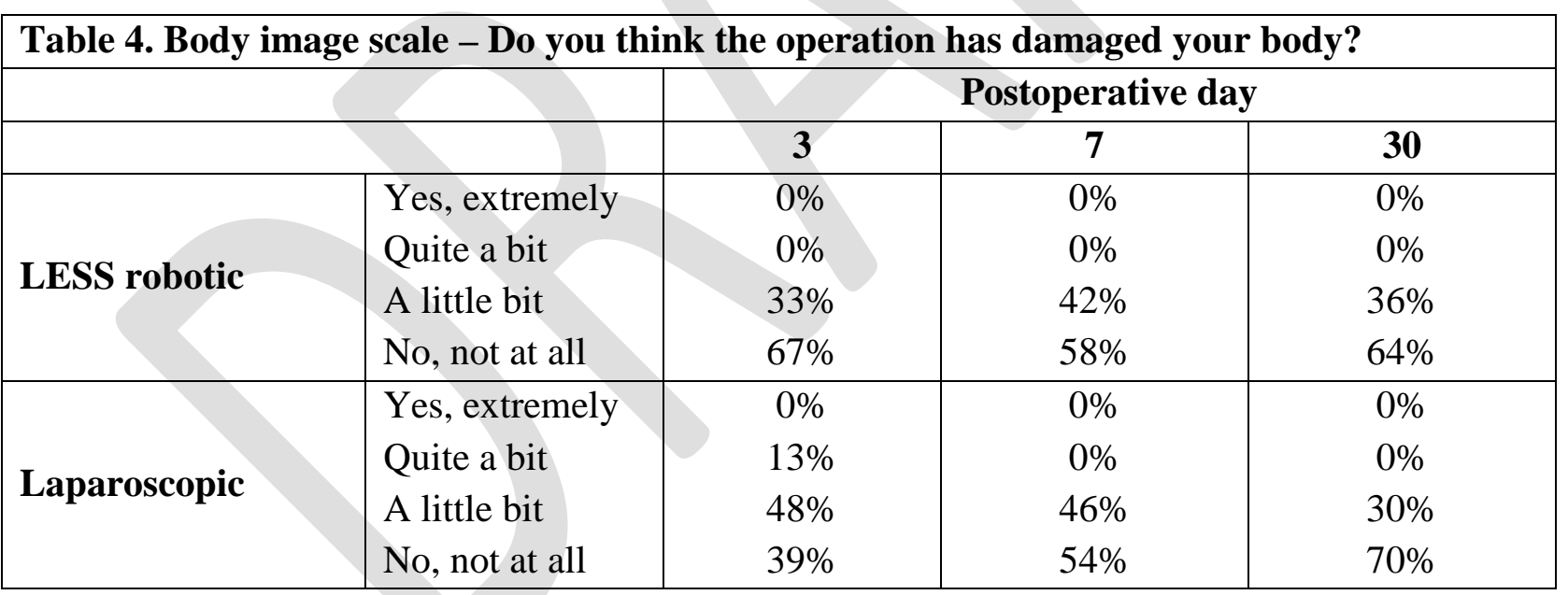

R-LESS group reported no perception of damage to their body on day 3 and 7 compared to LLDN group ( $\mathrm{p}=0.001)$; however by day 30 , both groups reported similar perception $(\mathrm{p}=0.51)$. LLDN: laparoscopic living donor nephrectomy; R-LESS: robotic laparoendoscopic single-site. 
Laparoendoscopic single-site vs. standard living donor nephrectomy

\begin{tabular}{|c|c|c|c|c|}
\hline & & \multicolumn{3}{|c|}{ Postoperative day } \\
\hline & & 3 & 7 & 30 \\
\hline LESS robotic & $\begin{array}{l}\text { Yes, extremely } \\
\text { Quite a bit } \\
\text { A little bit } \\
\text { No, not at all }\end{array}$ & $\begin{array}{c}0 \% \\
0 \% \\
18 \% \\
82 \%\end{array}$ & $\begin{array}{c}0 \% \\
4 \% \\
7 \% \\
89 \%\end{array}$ & $\begin{array}{c}0 \% \\
0 \% \\
0 \% \\
100 \%\end{array}$ \\
\hline Laparoscopic & $\begin{array}{l}\text { Yes, extremely } \\
\text { Quite a bit } \\
\text { A little bit } \\
\text { No, not at all }\end{array}$ & $\begin{array}{c}0 \% \\
4 \% \\
7 \% \\
89 \%\end{array}$ & $\begin{array}{l}0 \% \\
0 \% \\
8 \% \\
92 \%\end{array}$ & $\begin{array}{c}0 \% \\
0 \% \\
13 \% \\
87 \%\end{array}$ \\
\hline
\end{tabular}

Both groups reported similar responses to this question at all-time points on postoperative days 3 , 7, and 30, respectively ( $\mathrm{p}=0.41)$. LESS: laparoendoscopic single-site.

\begin{tabular}{|l|l|c|c|c|}
\hline \multicolumn{2}{|c|}{ Table 6. Cosmesis scale - On a scale of 1-7, how satisfied are you with your scar? } \\
\hline \multicolumn{3}{|c|}{ Postoperative day } \\
\hline \multirow{5}{*}{ LESS robotic } & 1 , Very unsatisfied & 3 & 7 & 30 \\
& 2 & $0 \%$ & $8 \%$ & $9 \%$ \\
& 3 & $0 \%$ & $0 \%$ & $0 \%$ \\
& 4 & $0 \%$ & $0 \%$ & $9 \%$ \\
& 4 & $0 \%$ & $17 \%$ & $9 \%$ \\
& 5 & $23 \%$ & $17 \%$ & $18 \%$ \\
& 6 & $23 \%$ & $8 \%$ & $0 \%$ \\
& 7, Very satisfied & $54 \%$ & $50 \%$ & $55 \%$ \\
\hline \multirow{5}{*}{ Laparoscopic } & 1, Very unsatisfied & $0 \%$ & $0 \%$ & $4 \%$ \\
& 2 & $0 \%$ & $12 \%$ & $0 \%$ \\
& 3 & $4 \%$ & $4 \%$ & $0 \%$ \\
& 4 & $31 \%$ & $24 \%$ & $13 \%$ \\
& 5 & $11 \%$ & $24 \%$ & $17 \%$ \\
& 6 & $27 \%$ & $0 \%$ & $31 \%$ \\
& 6 & $27 \%$ & $36 \%$ & $35 \%$ \\
\hline
\end{tabular}

R-LESS group were more satisfied with the cosmetic outcome of the surgery compared to the LLDN group on postoperative days 3, 7, and 30 ( $p=0.008$ ). LLDN: laparoscopic living donor nephrectomy; R-LESS: robotic laparoendoscopic single-site. 
Laparoendoscopic single-site vs. standard living donor nephrectomy

\begin{tabular}{|l|l|c|c|c|c|}
\hline \multicolumn{5}{|c|}{ Table 7. Visual analog pain scale } \\
\multicolumn{2}{|l|}{} & \multicolumn{4}{|c|}{ Postoperative day } \\
\hline \multirow{3}{*}{ LESS robotic } & $\mathbf{1}$ & $\mathbf{3}$ & $\mathbf{7}$ & $\mathbf{3 0}$ \\
& None (0) & $7 \%$ & $7 \%$ & $50 \%$ & $67 \%$ \\
& Mild (1-3) & $43 \%$ & $43 \%$ & $41 \%$ & $33 \%$ \\
& Moderate (4-6) & $35 \%$ & $33 \%$ & $8 \%$ & $0 \%$ \\
& Severe (7-10) & $14 \%$ & $14 \%$ & $0 \%$ & $0 \%$ \\
\hline \multirow{3}{*}{ Laparoscopic } & None (0) & $4 \%$ & $0 \%$ & $38 \%$ & $64 \%$ \\
& Mild (1-3) & $22 \%$ & $59 \%$ & $47 \%$ & $36 \%$ \\
& Moderate (4-6) & $52 \%$ & $26 \%$ & $15 \%$ & $0 \%$ \\
& Severe (7-10) & $22 \%$ & $15 \%$ & $0 \%$ & $0 \%$ \\
\hline
\end{tabular}

R-LESS group reported lower pain scores (none/mild pain R-LESS 50\% vs. 26\% LLDN group) on day 1 and 3 ( $\mathrm{p}<0.001)$; however from day 7 onwards, both groups showed similar perceptions of pain ( $\mathrm{p}=0.16)$. LLDN: laparoscopic living donor nephrectomy; R-LESS: robotic laparoendoscopic single-site. 